\title{
ANTICHAIN CUTSETS OF STRONGLY CONNECTED POSETS
}

\author{
STEPHAN FOLDES AND RUSS WOODROOFE
}

\begin{abstract}
Rival and Zaguia showed that the antichain cutsets of a finite Boolean lattice are exactly the level sets. We show that a similar characterization of antichain cutsets holds for any strongly connected poset of locally finite height. As a corollary, we characterize the antichain cutsets in semimodular lattices, supersolvable lattices, Bruhat orders, locally shellable lattices, and many more. We also consider a generalization to strongly connected $d$-uniform hypergraphs.
\end{abstract}

\section{INTRODUCTION}

An antichain cutset in a poset $P$ is a set of elements that intersects each maximal chain in exactly one element. For example, in a graded poset, any level set (consisting of all elements of a specified rank) is an antichain cutset. Rival and Zaguia showed [18, Theorem 4] that for a finite Boolean lattice the converse holds: every antichain cutset is a level set. Other papers discussing antichain cutsets include 2, 16, 11, 13, 19]; antichain cutsets also appear in Rota's well-known Crosscut Theorem [8, 24].

The purpose of the current paper is to generalize this converse result of Rival and Zaguia to wide families of finite and infinite posets. A poset $P$ is said to be strongly connected if for any two maximal chains $\mathbf{c}$ and $\mathbf{d}$ there is a sequence of maximal chains

$$
\mathbf{c}=\mathbf{c}^{(0)}, \mathbf{c}^{(1)}, \mathbf{c}^{(2)}, \ldots, \mathbf{c}^{(n)}=\mathbf{d}
$$

such that the symmetric difference of $\mathbf{c}^{(i)}$ and $\mathbf{c}^{(i+1)}$ has cardinality two. Then:

Acknowledgement: The work of the first-named author has been co-funded by Marie Curie Actions, and supported by the National Development Agency (NDA) of Hungary and the Hungarian Scientific Research Fund (OTKA) within a project hosted by the University of Miskolc, Department of Analysis. The work was also completed as part of the TAMOP-4.2.1.B10/2/KONV-2010-0001 project at the University of Miskolc, with support from the European Union, co-financed by the European Social Fund.

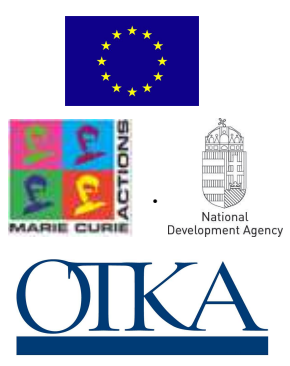


Theorem 1. If a poset $P$ is strongly connected with distinct antichain cutsets $A$ and $B$, then $A$ and $B$ are disjoint.

Theorem 1 will follow immediately from the somewhat stronger Theorem 5. We note that Theorem 11 holds for any poset, with no assumption that the poset is discrete or graded.

In the case of a discrete poset we can say more:

Theorem 2. If a discrete poset $P$ is strongly connected, then the antichain cutsets of $P$ are exactly the level sets.

We contrast Theorem 2 with the result of Behrendt 2 that any lattice may be obtained as the "lattice of antichain cutsets" of a poset with height 3. In the situation of Theorem 2, the lattice of antichain cutsets is a chain.

The term "strongly connected" comes from a relationship with geometry, and from the geometric combinatorics literature we obtain a large list of examples:

Proposition 3. Let $P$ be any locally finite height semimodular lattice or supersolvable lattice, or a Bruhat order of any Coxeter group, or any graded EL-shellable/shellable/Cohen-Macaulay poset. Then the antichain cutsets of $P$ are exactly the level sets.

The paper is organized as follows. In the remainder of this section, we introduce additional useful terminology for posets. In Section 2 we prove Theorems 1 and 2. In Section 3 we make the connection with geometric combinatorics, and prove Proposition 3. We close in Section 4 by briefly sketching a generalization to uniform hypergraphs.

The paper incorporates and expands upon [12].

1.1. Terminology and notation. For any poset $P$, the length of a finite chain $\mathbf{c}$ is one less than the cardinality of $\mathbf{c}$. The height of $P$ is the supremum of lengths over all finite chains in $P$.

Let $\mathcal{X}$ be some poset property, such as finiteness or strong connectivity. We say that $P$ is locally $\mathcal{X}$ if every interval $[a, b]$ in $P$ has property $\mathcal{X}$. For example, a poset is locally strongly connected if every interval is strongly connected, and has locally finite height if every interval has finite height.

We say that $P$ is pairwise-locally $\mathcal{X}$ if for all $x, y \in P$ there is some interval containing $x$ and $y$ that has property $\mathcal{X}$. It is obvious that if $\mathcal{X}$ is a property closed under taking subintervals, then locally $\mathcal{X}$ and pairwise-locally $\mathcal{X}$ are equivalent for lattices, but this does not hold for general posets. We remark that pairwise-locally $\mathcal{X}$ already requires that any $x$ and $y$ have some upper and lower bound. 
A poset is bounded if it has a greatest element $\hat{1}$ and least element $\hat{0}$. If $x<y$, and if there is no $z$ with $x<z<y$, then we write $x \lessdot y$, and say that $y$ covers $x$. If $P$ has a least element $\hat{0}$, then the atoms of $P$ are the elements covering $\hat{0}$.

Example 4. Consider the poset $E$ consisting of the natural numbers $\{1,2,3,4,5,30,25,200,300,600\}$, ordered by divisibility. It is easy to verify that $E$ is strongly connected, and since $E$ is bounded, it is also pairwise-locally strongly connected. On the other hand, the interval $[1,200]$ is not strongly connected, so $E$ is not locally strongly connected.

For any totally ordered set $R$, we define an $R$-grading or $R$-ranking of a poset $P$ to be a map $\rho: P \rightarrow R$ such that $\rho$ restricts on each maximal chain of $P$ to an isomorphism (onto $R$ ). In a poset with finite height $n-1$, the existence of an $R$-grading (or more specifically an $[n]$-grading, where $[n]=\{1, \ldots, n\}$ ) is obviously equivalent to the usual notion of gradedness as defined in e.g. [23]. More generally, a similar equivalence holds in any poset with all nonempty chains having a minimal element. A level set of a poset with an $R$-grading $\rho$ is any non-empty set of the form $L_{r}=\{x: \rho(x)=r\}$.

A poset is discrete if every interval has a maximal chain of finite length: thus, in a discrete poset $x<y$ if and only if there is a chain of cover relations between the two. We caution that the definition of discreteness for posets is not entirely consistent in the literature, and that for example 24] defines discreteness to mean locally finite height, a strictly stronger condition. This inconsistency should not be confusing, as we will mostly consider $R$-graded posets, where discreteness and locally finite height are equivalent.

For other standard poset notation and terminology, we refer to [23] or [24].

\section{Proof of Main Theorems}

We prove a stronger variant of Theorem 1, which follows by adding a $\hat{0}$ and $\hat{1}$ element if necessary:

Theorem 5. If $P$ is a pairwise-locally strongly connected poset with antichain cutsets $A$ and $B$, then $A$ and $B$ are disjoint.

Proof. Suppose by contradiction that $A$ and $B$ are antichain cutsets with nontrivial intersection. If $y \in A \cap B$ and $z \in B \backslash A$, then there is some strongly connected interval $I$ containing $y$ and $z$, and it follows easily that $A \cap I$ and $B \cap I$ are antichain cutsets for $I$.

Let $\mathcal{M}$ be the set of maximal chains on $I$ that intersect $B$ at a member of $A \cap B$, and $\mathcal{N}$ be the set of maximal chains on $I$ that 
intersect $B$ at a member of $B \backslash A$. By construction both $\mathcal{M}$ and $\mathcal{N}$ are nonempty. Applying the strongly connected property to $\mathbf{c} \in \mathcal{M}$ and $\mathbf{d} \in \mathcal{N}$ gives that there exist maximal chains $\mathbf{m} \in \mathcal{M}$ and $\mathbf{n} \in \mathcal{N}$ such that the symmetric difference of $\mathbf{m}$ and $\mathbf{n}$ is 2 .

Let $x$ be the unique element of $\mathbf{m} \cap A=\mathbf{m} \cap B$, and let $a, b$ denote respectively the unique elements of $\mathbf{n} \cap A$ and $\mathbf{n} \cap B=\mathbf{n} \cap(B \backslash A)$. But then $\mathbf{m} \backslash\{x\}$ intersects neither $A$ nor $B$, hence all of $x, a, b$ are contained in the symmetric difference of $\mathbf{m}$ and $\mathbf{n}$. It follows that $a=b$, a contradiction.

In the presence of an $R$-grading, we additionally have:

Theorem 6. If $P$ is a pairwise-locally strongly connected and $R$-graded poset (for some total order $R$ ), then the antichain cutsets of $P$ are exactly the level sets.

Proof. The level sets partition $P$, hence every antichain cutset intersects some level set. Since a level set is an antichain cutset (by definition of $R$-grading), the result follows from Theorem 5 ,

To prove Theorem 2 it remains to show that every discrete strongly connected poset is $R$-graded for some $R$. We start with a lemma:

Lemma 7. If $\mathbf{m}$ and $\mathbf{n}$ are maximal chains in a strongly connected poset $P$, then $\mathbf{m} \backslash \mathbf{n}$ and $\mathbf{n} \backslash \mathbf{m}$ have the same finite cardinality.

Proof. By strong connectivity, $\mathbf{m}$ and $\mathbf{n}$ differ by a finite number of exchanges; each exchange alters the cardinality of $|\mathbf{m} \backslash \mathbf{n}|$ and $|\mathbf{n} \backslash \mathbf{m}|$ by the same number $(0,1$, or -1$)$.

Corollary 8. If $P$ is a discrete poset which is strongly connected, locally strongly connected, or pairwise-locally strongly connected, then every interval of $P$ is graded of finite height.

Lemma 9. Let $P$ be a discrete and locally graded poset such that every pair of elements $x, y \in P$ have an upper bound and a lower bound in common. Then $P$ is $R$-graded for some $R \subseteq \mathbb{Z}$.

Proof. Pick some $x_{0} \in P$ and define $\rho\left(x_{0}\right)=0$. Then for any $y \in P$, there is a lower bound $w$ and an upper bound $z$ of $x_{0}$ and $y$. We let $\rho(y)=\operatorname{height}[w, y]-$ height $\left[w, x_{0}\right]$. It is clear that $\rho$ is injective on each maximal chain of $P$, and any two maximal chains are mapped by $\rho$ onto the same subset of $\mathbb{Z}$ by the existence of common upper bounds.

It remains to check that $\rho$ is well-defined, i.e., that it doesn't depend on the choice of $w$. But if we pick some other lower bound $w^{\prime}$ of $x_{0}$ and $y$, then there is a lower bound $v$ of $w$ and $w^{\prime}$, and then $\rho$ restricts to a (well-defined) finite height grading of $[v, z]$. 
Corollary 10. If $P$ is a discrete poset which is strongly connected or pairwise-locally strongly connected, then $P$ is $R$-graded for some $R \subseteq \mathbb{Z}$.

Proof. For $P$ pairwise-locally strongly connected this is immediate by Lemma 9 with Corollary 8 .

For $P$ strongly connected, it is clear that all maximal chains are isomorphic. If some pair of elements $x$ and $y$ fail to have a common lower bound, then all maximal chains have a least element by an easy application of Lemma [7. Then $P \cup\{\hat{0}\}$ is discrete, and $\hat{0}$ is a lower bound for all elements. Similarly for upper bounds.

We augment $P$ by $\hat{0}$ and/or $\hat{1}$ if necessary (as in the preceding paragraph) to obtain a discrete poset $\widehat{P}$ where every pair of elements has a common upper and lower bound. Since adding/deleting $\hat{0}$ and/or $\hat{1}$ preserves strong connectivity, we obtain from Lemma 9 with Corollary 8 that $\widehat{P}$ is $R$-graded. The conclusion follows for $P$.

\section{Relationships With GEOMETRIC COMBinatorics}

3.1. Locally connected posets and their order complexes. The name "strongly connected" comes from a relationship with geometric combinatorics.

Recall that an (abstract) simplicial complex is a family of finite sets (called faces) on a base set (called vertices) which is closed under inclusion, and that any abstract simplicial complex can be treated as a topological space (specifically, a cell complex - see e.g. [14]) by identifying each face $\sigma$ having $(n+1)$ points with an $n$-dimensional simplex. The dimension of a simplicial complex is the supremum over all faces of the dimension of this corresponding simplex.

A finite dimensional simplicial complex is strongly connected if for every pair of facets (maximal faces) $\sigma$ and $\tau$, there is a sequence of facets between $\sigma$ and $\tau$ with each adjacent pair having symmetric difference 2. For example, any simplicial complex realizing a manifold is strongly connected.

Associated with any finite height poset $P$ is a simplicial complex $\Delta(P)$ (the order complex), where the vertices are the elements of $P$, and the faces consist of all chains in $P$ 25]. The poset properties of $P$ are closely related to the topological properties of $\Delta(P)$ : for example, the Möbius number $\mu_{P}(x, y)$ of a finite interval $[x, y]$ is exactly the reduced Euler characteristic $\tilde{\chi}(\Delta(x, y))$. We remark that bounded posets are contractible (such a poset $P$ is a topological cone over $\Delta(P \backslash\{\hat{0}\})$ ), so it is natural to consider open intervals or $\Delta(P \backslash\{\hat{0}, \hat{1}\})$. 
In particular, it is clear that a finite height poset $P$ is strongly connected in our sense if and only if $\Delta(P)$ is strongly connected in the simplicial complex sense.

We say that a poset $P$ is connected if there is a sequence $x=x_{0} \leq$ $x_{1} \geq x_{2} \leq \ldots x_{n}=y$ between any two elements $x$ and $y$. In the finite height case, this is equivalent to $\Delta(P)$ being path-connected; and in the discrete case it is equivalent to the Hasse diagram being a connected graph. As before, a bounded poset or closed interval is always connected, so to obtain non-trivial statements we remove $\hat{0}$ and $\hat{1}$ or consider open intervals.

The following generalization of [8, Proposition 11.7 and following] (see also [15, Lemma 4.2]) to infinite posets is proved entirely similarly to the finite case:

Lemma 11. If $P$ is a bounded poset of finite height such that every open interval $(x, y)$ in $P$ of nonzero height is connected, then $P$ is strongly connected.

We see from Example 4 that the sufficient condition of Lemma 11 is not necessary. We further comment that the result of Lemma 11 fails to hold in posets with infinite height, as the following example demonstrates:

Example 12. Consider the lattice $L=(\mathbb{N} \times \mathbb{N}) \cup \hat{1}$. Every closed interval is isomophic to either $[n] \times[m]$ or to $L$ itself, hence every open interval of nonzero height is connected. But there is no strongly connected sequence interpolating between the chain $\{(a, 0): a \in \mathbb{N}\}$ and $\{(0, b): b \in \mathbb{N}\}$, as every interval of the form $\left[\left(a_{0}, 0\right),\left(a_{n}, 0\right)\right]$ is a chain.

We comment that since $\mathbb{N} \times \mathbb{N}$ is locally strongly connected and hence pairwise-locally strongly connected, the antichain cutsets of $L$ nonetheless consist exactly of the level sets.

Remark 13. Theorem 5 gives a connection between the combinatorics of antichain cutsets in $P$ and the topology of $\Delta(P)$. Bell and Ginsburg [3] (see also [13]) gave another topological condition, showing that a certain space $M(P)$ is compact if and only if every $x \in P$ is in some finite antichain cutset. We are not aware of any connection between the order complex and the topology considered by Bell and Ginsburg.

3.2. EL-labelings. An edge labeling of a locally finite height poset $P$ is a map $\lambda$ from the cover relations of $P$ (i.e., the edges of the Hasse diagram) to some partially ordered set $\Lambda$, usually the integers. Edge labelings associate a word of elements from $\Lambda$ with every maximal chain 
on an interval of $P$, and we order such chains lexicographically by their associated words. We say that $\lambda$ has an ascent at $y$ if $\lambda(x \lessdot y) \leq \lambda(y \lessdot z)$, and a descent otherwise; a chain is ascending if every element other than the top and bottom is an ascent.

An EL-labeling of a locally finite height poset $P$ is an edge labeling such that on every interval $[x, y]$ of $P$ :

(1) There is a unique ascending maximal chain $\mathbf{a}^{[x, y]}$.

(2) The ascending chain $\mathbf{a}^{[x, y]}$ lexicographically precedes all other maximal chains on $[x, y]$.

When $P$ is infinite we also require the following, which we note to be automatic in the finite case:

(3) The lexicographic order of maximal chains on $[x, y]$ has a linear extension which is a well-order.

Example 14. In a finite height semimodular lattice $L$, any (strict) well-ordering $\prec$ of the join irreducible elements such that $x<y$ implies $x \prec y$ induces an $E L$-labeling with label set $L$. The labeling assigns to $x \lessdot y$ the first join irreducible $z$ such that $x \vee z=y$ [21, Proposition $2.2]$.

A precursor to EL-labelings was introduced by Stanley [21] as a purely combinatorial description of some similar behavior between geometric lattices and subgroup lattices of supersolvable groups, and the definition as above was made and studied by Björner [6], and Björner and Wachs [10. The existence of an EL-labeling has strong consequences for the topology of $\Delta(P)$, and we recommend [25] for a highly readable account of this.

One such consequence is the following:

Lemma 15. If $P$ is a bounded poset which is graded of finite height and admits an EL-labeling, then $P$ is strongly connected.

Proof. It suffices to show that there is a sequence as in the definition of strong connectivity from any chain $\mathbf{c}$ to the unique ascending chain $\mathbf{a}^{[\hat{0}, \hat{1}]}$. Suppose that $\mathbf{c}=\left\{\hat{0}=y_{0} \lessdot y_{1} \lessdot \cdots \lessdot y_{n}=\hat{1}\right\}$ has a descent at $y_{i}$. Then applying the $E L$-labeling property on $\left[y_{i-1}, y_{i+1}\right]$ allows us to replace $y_{i}$ with a $y_{i}^{\prime}$ so that $\mathbf{c}^{\prime}=\left(\mathbf{c} \backslash y_{i}\right) \cup y_{i}^{\prime}$ has an ascent at $y_{i}^{\prime}$, and we notice that $\mathbf{c}$ and $\mathbf{c}^{\prime}$ have symmetric difference two. Since $\mathbf{c}^{\prime}$ lexicographically precedes $\mathbf{c}$, repeating this process leaves us with $\mathbf{a}^{[\hat{0}, \hat{1}]}$ after a finite number of steps (by the well-ordering condition).

Corollary 16. If $P$ is a locally finite height $R$-graded lattice with an $E L$-labeling, then the antichain cutsets of $P$ are exactly the level sets. 
Proof. Apply Lemma 15, then Theorem 6.

Remark 17. Although the proofs become less elementary, the consequence of Lemma 15 holds more generally for the class of CohenMacaulay posets, as discussed below in Section 3.3.

3.3. Shellable and Cohen-Macaulay complexes . We very briefly discuss some closely related properties implied by the existence of an EL-labeling. For further background, refer to [25] for finite posets, and [7, especially Remark 4.21] for the infinite (but finite height) case. Another related work [1] considers locally finite shellable posets from an algebraic point of view.

A finite height poset is shellable if there is a well-ordering $\prec$ (called a shelling) of the maximal chains of $P$ such that if $\mathbf{c} \prec \mathbf{d}$ then there is some $\mathbf{c}^{\prime} \prec \mathbf{d}$ with $\mathbf{c} \cap \mathbf{d} \subseteq \mathbf{c}^{\prime} \cap \mathbf{d}=\mathbf{d} \backslash\{x\}$ for some $x$. Every interval of a shellable poset is also shellable. For example, if $P$ is a bounded poset of finite height admitting an EL-labeling, then (a well-ordered linear extension of) the lexicographic order is a shelling. As a result, posets with an EL-labeling are often referred to as EL-shellable. The proof of Lemma 15 adapts straightforwardly to shellable posets.

A finite height poset is Cohen-Macaulay if it obeys a certain technical condition involving vanishing homology groups in low dimension for every interval - the details will not be important here, except to note that every graded shellable poset is Cohen-Macaulay. A CohenMacaulay poset has the property that every open interval of nonzero height is connected, hence by Lemma 11 a Cohen-Macaulay poset is strongly connected.

We summarize the chain of implications for a finite height graded poset:

bounded + admits EL-labeling $\Longrightarrow$ shellable $\Longrightarrow$ Cohen-Macaulay

$\Longrightarrow$ strongly connected,

and comment that each implication is strict.

Corollary 18. If $P$ is a Cohen-Macaulay poset (more generally, a pairwise-locally Cohen-Macaulay poset), then the antichain cutsets of $P$ are exactly the level sets.

3.4. Examples. There is a vast literature on finite or locally finite posets and lattices with EL-labelings and/or shellings, as we touch upon in Proposition 3 .

Example 19 (Discrete semimodular lattices). As previously mentioned, any finite height semimodular lattice admits an EL-labeling. Thus, a 
discrete (equivalently locally finite height) semimodular lattice has an $E L$-labeling in every interval, so is locally strongly connected. As such a lattice is well-known to be $R$-graded (for some $R \subseteq \mathbb{Z}$ ), the antichain cutsets of a discrete semimodular lattice are exactly the level sets. Thus the result of [12] is recovered.

As a special case, the lattice of flats of any matroid has antichain cutsets consisting exactly of its level sets.

The subgroup lattice $L(G)$ of a group $G$ consists of all subgroups of $G$, ordered by inclusion.

Example 20 (Supersolvable lattices). A finite height lattice is supersolvable if it has height $n$, and has an $E L$-labeling which labels every maximal chain with a permutation of $[n]$ [20, 17]. It follows that a supersolvable lattice is necessarily graded, and so has antichain cutsets consisting exactly of the level sets. Examples of supersolvable lattices include subgroup lattices of finite supersolvable groups, as well as partition lattices [20].

In particular, the subspace lattice of a finite vector space is both (semi-)modular and supersolvable. Hence we immediately obtain a $q$ analogue of [18, Theorem 4]: the antichain cutsets of the subspace lattice of any finite vector space are exactly the level sets.

We also obtain that the subgroup lattice of $\mathbb{Z}$ has antichain cutsets consisting exactly of its level sets, as $L(\mathbb{Z})$ is $\left(\mathbb{Z}^{-} \cup \hat{0}\right)$-graded and every interval of $L(\mathbb{Z}) \backslash\{\hat{0}\}$ is finite and supersolvable (and indeed distributive).

Example 21 (Bruhat order). Let $W$ be a Coxeter group (see [9]) generated by involutions $S$, and let $\ell(w)$ be the minimal length word expressing $w \in W$ as the product of generators from $S$. The Bruhat order associated with $(W, S)$ has a cover relation between $w$ and $w t$ if $t$ is conjugate to some $s \in S$ and $\ell(w t)=\ell(w)+1$. Bruhat orders have locally finite height [9, Corollary 2.2.4], are locally and pairwise-locally shellable [9, Proposition 2.2.9 and Corollary 2.7.5], and are graded by $\ell$, hence their antichain cutsets are exactly the level sets.

\section{Generalization to hypergraphs}

We remark briefly that our proof of Theorem 1 has a generalization to $d$-uniform hypergraphs. Recall that a transversal of a hypergraph is a subset $S$ of vertices that has nonempty intersection with every edge. General background on transversals can be found in [4]. An exact transversal is a transversal that meets each edge in exactly one vertex. Thus, an antichain cutset for a poset $P$ is an exact transversal 
for the hypergraph with vertex set $P$ and edge set consisting of the maximal chains of $P$.

We define a $d$-uniform hypergraph $\mathcal{H}$ to be strongly connected if for every pair $e$ and $f$ of edges of $\mathcal{H}$ there is a sequence

$$
e=e^{(0)}, e^{(1)}, \ldots, e^{(n)}=f
$$

such that every adjacent pair has symmetric difference 2 . The simplicial complex $\langle\mathcal{H}\rangle$ generated by $\mathcal{H}$ has faces consisting of all subsets of any edge of $\mathcal{H}$. It is clear that $\mathcal{H}$ is strongly connected if and only if $\langle\mathcal{H}\rangle$ is strongly connected in the simplicial complex sense discussed in Section 3.1 .

The argument leading to Theorem 1 translates straightforwardly to:

Theorem 22. If $\mathcal{H}$ is a strongly connected d-uniform hypergraph, then the exact transversals of $\mathcal{H}$ are pairwise disjoint.

The definitions for shellability and Cohen-Macaulay that we presented in Section 3.3 are specialized from more general definitions of simplicial complexes, and strong connectivity still holds for such.

Corollary 23. If $\langle\mathcal{H}\rangle$ is pure shellable or Cohen-Macaulay, then the exact transversals of $\mathcal{H}$ are pairwise disjoint.

A $d$-uniform hypergraph is balanced if there is a $d$-coloring of the vertices such that no edge contains two vertices with the same color. Balanced uniform hypergraphs generalize (the set of maximal chains of) graded posets. There is some literature on balanced Cohen-Macaulay simplicial complexes [22, Chapter III.4 and references].

The same proof as that of Theorem 6 yields:

Theorem 24. If $\mathcal{H}$ is a balanced strongly connected d-uniform hypergraph, then the exact transversal sets of $\mathcal{H}$ consist exactly of the color classes of vertices.

We comment that Theorems 24 and 2 can be seen as a generalization of the fact that a connected bipartite graph has a unique 2-coloring (up to renaming the colors).

Example 25. Consider the chessboard complex: the simplicial complex with vertex set the squares of an $a \times b$ chessboard $(a \leq b)$, and faces consisting of "non-taking rook configurations", i.e., sets of squares with no two on the same row or column. For any $a, b$ this simplicial complex is clearly $(a-1)$-dimensional. Let $\mathcal{C}_{a, b}$ be the hypergraph of maximal faces, that is, with edges consisting of sets of $a$ non-taking rooks.

We notice that $\mathcal{C}_{a, b}$ is balanced, since every edge contains a rook from each row. If $a<b$, then it is straightforward to show by exchanging 
rooks that $\mathcal{C}_{a, b}$ is strongly connected (this was essentially observed in [5]). It follows that if $a<b$ then the exact transversals for $\mathcal{C}_{a, b}$ are in bijection with the rows of the chessboard, and consist of $b$ vertices corresponding to the squares of the given row.

In contrast, we notice that $\mathcal{C}_{a, a}$ does not have disjoint exact transversals, since we have exact transversals in correspondence with the rows of the chessboard, and (by transposition) additional exact transversals in correspondence with the columns.

\section{REFERENCES}

[1] Annetta Aramova, Jürgen Herzog, and Takayuki Hibi, Shellability of semigroup rings, Nagoya Math. J. 168 (2002), 65-84.

[2] Gerhard Behrendt, The lattice of antichain cutsets of a partially ordered set, Discrete Math. 89 (1991), no. 2, 201-202, 10.1016/0012-365X(91)90368-C.

[3] Murray Bell and John Ginsburg, Compact spaces and spaces of maximal complete subgraphs, Trans. Amer. Math. Soc. 283 (1984), no. 1, 329-338, $10.2307 / 2000007$.

[4] Claude Berge, Hypergraphs, North-Holland Mathematical Library, vol. 45, North-Holland Publishing Co., Amsterdam, 1989, Combinatorics of finite sets, Translated from the French.

[5] A. Björner, L. Lovász, S. T. Vrećica, and R. T. Živaljević, Chessboard complexes and matching complexes, J. London Math. Soc. (2) 49 (1994), no. 1, 25-39.

[6] Anders Björner, Shellable and Cohen-Macaulay partially ordered sets, Trans. Amer. Math. Soc. 260 (1980), no. 1, 159-183.

[7] _ Some combinatorial and algebraic properties of Coxeter complexes and Tits buildings, Adv. in Math. 52 (1984), no. 3, 173-212, 10.1016/00018708(84)90021-5.

[8] _ Topological methods, Handbook of combinatorics, Vol. 1, 2, Elsevier, Amsterdam, 1995, pp. 1819-1872.

[9] Anders Björner and Francesco Brenti, Combinatorics of Coxeter groups, Graduate Texts in Mathematics, vol. 231, Springer, New York, 2005.

[10] Anders Björner and Michelle L. Wachs, Shellable nonpure complexes and posets. I, Trans. Amer. Math. Soc. 348 (1996), no. 4, 1299-1327.

[11] Mohamed H. El-Zahar and Nejib Zaguia, Antichains and cutsets, Combinatorics and ordered sets (Arcata, Calif., 1985), Contemp. Math., vol. 57, Amer. Math. Soc., Providence, RI, 1986, pp. 227-261.

[12] Stephan Foldes, Note on antichain cutsets in discrete semimodular lattices, 2011, arXiv:1102.5456.

[13] J. Ginsburg, I. Rival, and B. Sands, Antichains and finite sets that meet all maximal chains, Canad. J. Math. 38 (1986), no. 3, 619-632, 10.4153/CJM1986-031-3.

[14] Allen Hatcher, Algebraic topology, Cambridge University Press, Cambridge, 2002.

[15] Steven Klee, The fundamental group of balanced simplicial complexes and posets, Electron. J. Combin. 16 (2009), no. 2, Special volume in honor of Anders Bjorner, Research Paper 7, 12. 
[16] Bo Yu Li, Finite antichain cutsets in posets, Acta Math. Sinica (N.S.) 7 (1991), no. 1, 51-61, A Chinese summary appears in Acta Math. Sinica 35 (1992), no. $2,287$.

[17] Peter McNamara and Hugh Thomas, Poset edge-labellings and left modularity, European Journal of Combinatorics 27 (2006), no. 1, 101-113, arXiv:math.CO/0211126.

[18] Ivan Rival and Nejib Zaguia, Antichain cutsets, Order 1 (1985), no. 3, 235-247, 10.1007/BF00383600.

[19] _ Effective constructions of cutsets for finite and infinite ordered sets, Acta Sci. Math. (Szeged) 51 (1987), no. 1-2, 191-207.

[20] Richard P. Stanley, Supersolvable lattices, Algebra Universalis 2 (1972), 197217.

[21] , Finite lattices and Jordan-Hölder sets, Algebra Universalis 4 (1974), 361-371.

[22] _ Combinatorics and commutative algebra, second ed., Progress in Mathematics, vol. 41, Birkhäuser Boston Inc., Boston, MA, 1996.

[23] _ Enumerative combinatorics. Vol. 1, Cambridge Studies in Advanced Mathematics, vol. 49, Cambridge University Press, Cambridge, 1997, With a foreword by Gian-Carlo Rota, Corrected reprint of the 1986 original.

[24] Manfred Stern, Semimodular lattices, Encyclopedia of Mathematics and its Applications, vol. 73, Cambridge University Press, Cambridge, 1999, 10.1017/CBO9780511665578, Theory and applications.

[25] Michelle L. Wachs, Poset topology: Tools and applications, Geometric combinatorics, IAS/Park City Math. Ser., vol. 13, Amer. Math. Soc., Providence, RI, 2007, arXiv:math/0602226, pp. 497-615.

Department of Mathematics, Tampere University of Technology, PL 553, 33101 TAMPERE, Finland

E-mail address: sf@tut.fi

Department of Mathematics, Washington University in St. Louis, St. Louis, MO, 63130

E-mail address: russw@math.wustl.edu 